\title{
TUMOR REGION EXTRACTION USING EDGE DETECTION METHOD IN BRAIN MRI IMAGE
}

\author{
Oniza Shaikh \\ Department of EXTC \\ ARMIET, Shahapur, Maharashtra, India
}

\begin{abstract}
The segmentation of the magnetic resonance imaging (MRI) of the brain is a complex problem in the field of medical imaging despite the various methods presented. The MRI image of the human brain can be divided into several subregions, especially in soft tissues such as gray matter, white matter and fluid. The combinatorial algorithm provides a solution to overcome the associated challenges of segmented brain MRI. The analysis of asymmetry of the brain is of great importance because not only is it an indicator of brain cancer, but it also predicts a future potential risk for it. In our work, we have concentrated to segment the anatomical region of the brain, divide the two halves of the brain and detect each half to detect the presence of a tumor. The bilateral and mathematical analysis with the Laplacian, gradient operator operates on real images, and the results show that the algorithm is flexible and convenient.
\end{abstract}

Keywords- MRI brain, asymmetry analysis, Bilateral Symmetry anatomical, Laplacian, Gradient.

\section{INTRODUCTION}

\subsection{Problem definition}

There are number of techniques to segment an image into region that are homogeneous or discontinuity. Not all the techniques are suitable for medical image analysis because of complexity and inaccuracy. There is no standard image segmentation technique that can produce satisfactory for all imaging application like brain, MRI .brain cancer diagnosis etc. Optimal selection of features, tissues, brain and non-brain element are considered as main obstacle for brain image segmentation. Accurate segmentation over full field of view is another hindrance .Operator supervisor and manual thresholding are other barriers to segment brain image. During the image segmentation procedure verification of result is another source of difficulty.

\subsection{Objective}

Objective to use more meaningful information to improve the segmentation and extraction of brain tumors

Approach An approach that uses bilateral symmetry information as an additional feature for segmentation

\author{
Kailash Jadhao \\ Department of EXTC \\ ARMIET, Shahapur, Maharashtra, India
}

Analysis Mathematical analysis increases performance to improve general automatic segmentation and brain tumor removal systems

If the incoming brain image is colored, it becomes a gray image. First read the red, blue and green value of each pixel and then, after the formulation, three different values will be converted to gray value. The automatic edge detection technique is proposed to automatically detect the edges of regions of interest in digital images. The method is used to segment an image into two symmetric regions based on the search for pixels that are similar in nature. The more symmetrical the two regions have, the more the edges weaken. At the same time, the non-symmetrical edges are improved. At the end, according to the effect of improvement, asymmetric regions can be detected, which is caused by a brain tumor.

\section{LITERATURE SURVEY}

\subsection{Magnetic resonance imaging (MRI)}

Magnetic resonance imaging (MRI) is an imaging technique based on the physical phenomenon of Nuclear Magnetic Resonance (NMR). It is used in medical environments to produce images of the interior of the human body. MRI can produce an image of the NMR signal in a thin slice through the human body. By scanning a set of such cuts, a volume of a part of the human body can be represented by MRI

\subsection{Image Segmentation Methods}

We review primarily those studies that are based on finding object regions in grey-level images. We also mention couple of studies that deal with color segmentation to highlight how this has been used for outdoor scene analysis. Image segmentation has been approached from a wide variety of perspectives. Our summary is presented for histogram thresholding, edge based segmentation, tree/graph based approaches, region growing, clustering, and probabilistic or Bayesian approaches, neural networks for segmentation.

\subsection{Proposed system}

It is based on the method of image segmentation, which refers to the main step in the processing of images, the entries are 


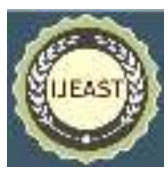

images and the outputs are the attributes extracted from those images. It will help to discover the symmetrical extraction of the brain image.

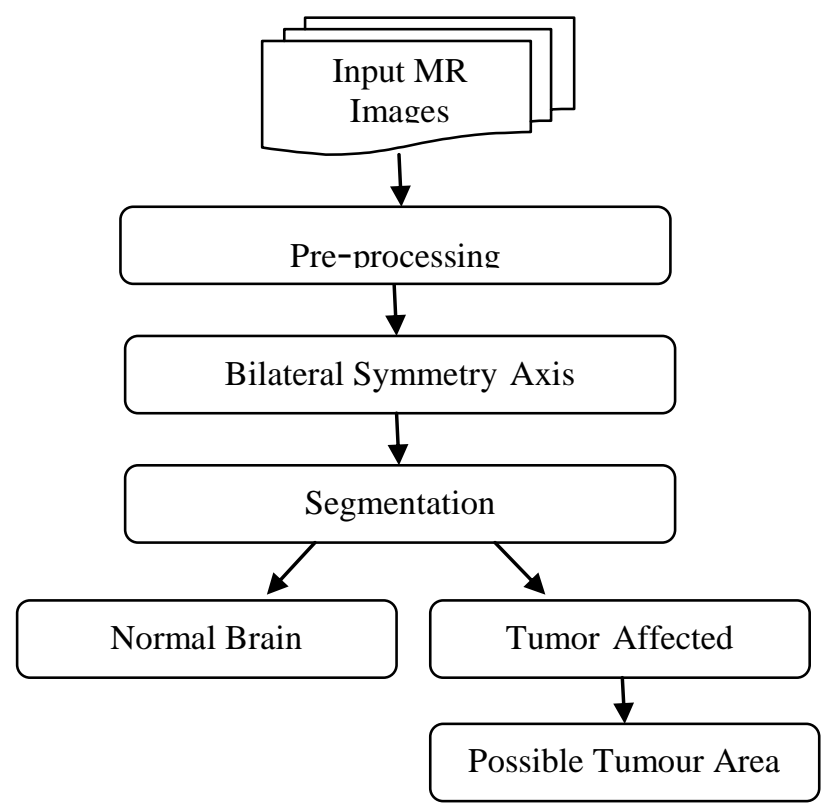

\subsection{Data preprocessing}

MRI images are subject to various types of noise, such as irregularities, etc. These noises can degrade the quality of the MR image and, therefore, can not provide correct information for subsequent image segmentation and edge detection. In order to improve the quality of the MR image, operations must be performed to eliminate or diminish the impairments suffered in its acquisition. Pre-processing is also needed to homogenize and separate the intensity. Distributions of malignant and benign tissues. This can be achieved using several demonization techniques, namely, Gaussian filter, median filter.

\subsubsection{Bilateral symmetry axis}

The definition of the axis of bilateral symmetry is a simple evaluation method that is commonly used to compare the corresponding MRI brain images to determine the ROI in the image. The structural and functional asymmetry in the human brain and nervous system is reviewed in a historical perspective. Brain asymmetry is one of those examples, which is a difference in size or shape, or both. The analysis of asymmetry of the brain is of great importance because not only is it an indicator of brain cancer, but it also predicts a future potential risk for it. In our work, we have concentrated to segment the anatomical regions of the brain, isolate the edge of each of them to investigate the presence of asymmetry of anatomical regions in MRI. The term asymmetry is often replaced by the term laterality when it comes to differences from left to right in psychology and neurosciences. However, although the term asymmetry can mean differences from left to right, both structural and functional, laterality is generally only used in relation to functional asymmetry.

\section{METHDOLOGY}

\subsection{Algorithm}

Step 1: - The edge detection method can be used to find the edges in the incoming brain image. It consists of five steps

- Smoothing: - blur the image to eliminate the noise present in the image

- Gradient search: - The edges should be marked where the gradients of the image have a large magnitude.

- No maximum suppression: -The local maxima should only be marked as border.

- Double threshold: -Permit potentials determined by the threshold.

- Edge tracking: - The final edges are determined by deleting all edges that are not connected to a very secure edge (strong)

Step 2: - Find the curve using LMS and the crammer rule.

Step 3: - The curve shows the area affected by the tumor.

Step 4: - Calculate the area affected by the tumor.

Step 5: - Extract the affected area from the brain image.

\section{PERFORMANCE ANALYSIS}

We have applied a proposed method for a segmentation of brain images. In this section we present the experimental result that indicates the different stages of the method.

\subsection{Experimental Analysis}

Several simulated experiments are carried out to demonstrate the validity and viability of the segmentation method to segment regions from images of the brain. The measures reflect the effectiveness of an image segmentation method. The system has been implemented using MATLAB due to its powerful mathematical and image processing functions.

In the first step, the color image is transformed from RGB to gray scale. Although, traditionally, RGB is the most used model for MRI images. All our data is obtained in the Phillips / Siemens / Wipro 1.5T scanners for the segmentation of brain images. Table 4.1 shows a detailed description of the patient with his disease and the grade that contains original MRI images with tumor used for this study. The input images are from patient identification 397384 (high grade) 19430618 (low grade) as shown in the figure. 6.1

Table 4.1: Database for segmentation

\begin{tabular}{|l|l|l|l|c|}
\hline $\begin{array}{l}\text { Patient } \\
\text { ID }\end{array}$ & Age & Gender & Disease & Grade \\
\hline 11 & 40 & Male & Oligodendroglia & High \\
\hline 22 & 70 & Male & $\begin{array}{l}\text { Metastatic } \\
\text { Deposits }\end{array}$ & High \\
\hline
\end{tabular}


International Journal of Engineering Applied Sciences and Technology, 2019

Vol. 4, Issue 3, ISSN No. 2455-2143, Pages 244-248

Published Online July 2019 in IJEAST (http://www.ijeast.com)

\begin{tabular}{|l|l|l|l|l|}
\hline 33 & 58 & Male & $\begin{array}{l}\text { Metastatic } \\
\text { Pathology }\end{array}$ & High \\
\hline 44 & 35 & Female & Mixed Glioma & Low \\
\hline 55 & 50 & Female & Cystic Glioma & \\
\hline
\end{tabular}

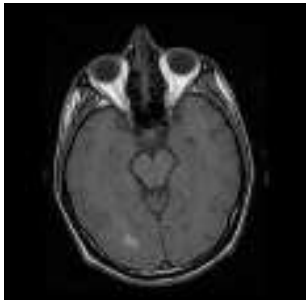

a. High Grade

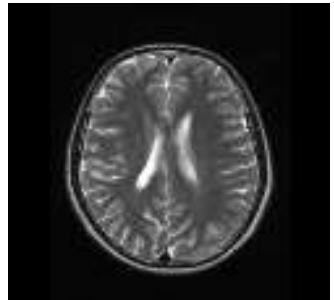

b. Low Grade

Figure 4.1: Input image from database

Next, we use the edge detection algorithm to segment a given input image. The following figure shows a different edge detected segmented image.

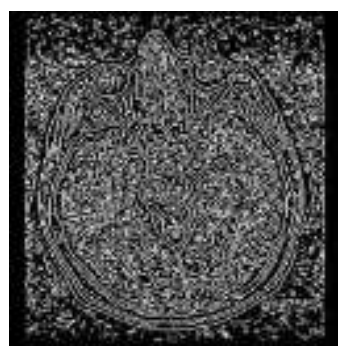

a. High Grade

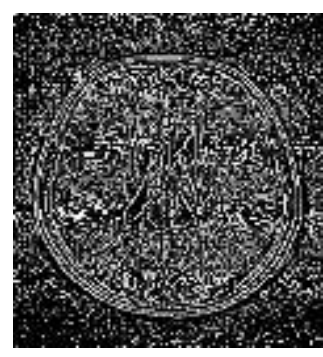

b. Low Grade

Figure 4.2: Edge detected by Robert filter

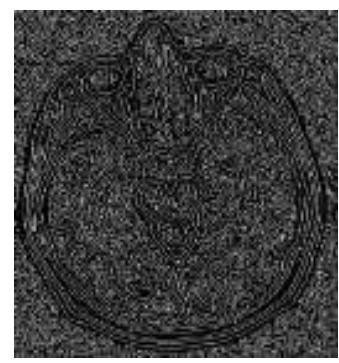

a. High Grade

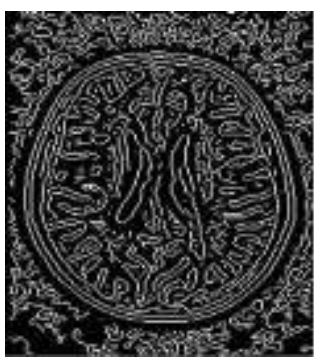

b. Low Grade

Figure 4.3: Edge detected by Prewitt filter

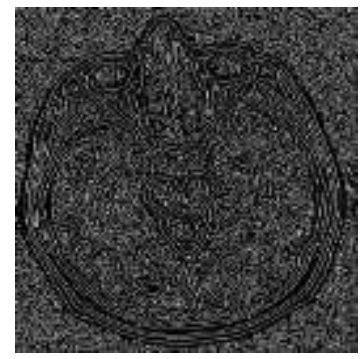

a. High Grade

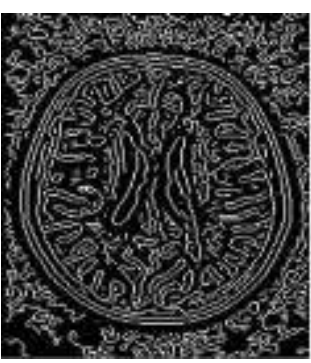

b. Low Grade
Figure 4.4: Edge detected by Canny filter

The previous figure shows the results of each edge detection algorithm for a given input image. The Roberts and Prewitt method finds edges using the Roberts and Prewitt approximation to the derivative, respectively.

Table 4.2: Number of detected edges

\begin{tabular}{|c|l|l|l|l|}
\hline Patient ID & Grade & \multicolumn{3}{|c|}{ No. of detected Edges } \\
\cline { 3 - 5 } & & Robert & Prewitt & Canny \\
\hline 101 & High & 6259 & 4382 & 1997 \\
\hline 102 & High & 7120 & 4323 & 2302 \\
\hline 103 & High & 6807 & 5757 & 1836 \\
\hline
\end{tabular}

The evaluation of the images showed that in noisy conditions, Canny, Prewitt, Robert, exhibit better performance, respectively. Canny gave the best results as shown in Figure 4.4. This was expected as Canny edge detection accounts for regions in an image. Canny produces fine lines for its edges by using no maximum suppression. Canny also uses hysteresis with threshold

During the processing of the image, the edge information is the main clue in the segmentation of the image. But, unfortunately, you can not get a better result in analyzing the content of the images without combining other information. Then, we combine the information of edges with the axis of bilateral symmetry to improve the effect of segmentation.

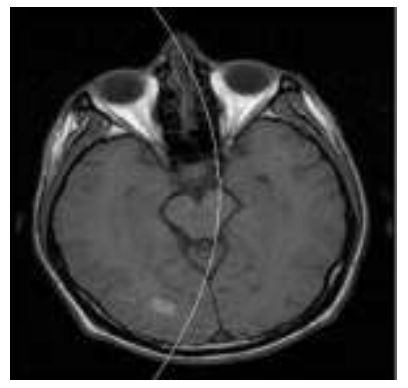

a. High Grade

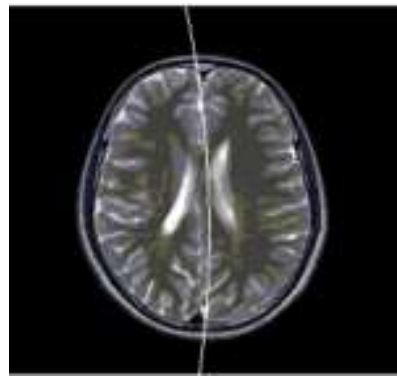

b. Low Grade
Figure 4.5: Bilateral Axis 
International Journal of Engineering Applied Sciences and Technology, 2019

Vol. 4, Issue 3, ISSN No. 2455-2143, Pages 244-248

Published Online July 2019 in IJEAST (http://www.ijeast.com)

Now to detect the position and the limit of the tumors automatically determined entry. The more symmetrical the two regions have, the more the edges weaken. At the same time, the non-symmetrical edges are improved. At the end, according to the effect of improvement, asymmetric regions can be detected, which is caused by a brain tumor. The possible tumor area is given input as shown in Figure 4.6

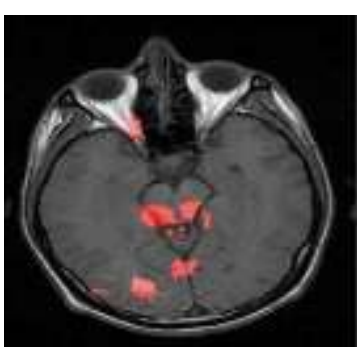

a. High Grade

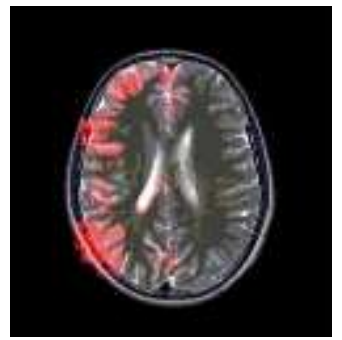

b. Low Grade
Figure 4.6: Possible tumor area

Table 4.3: Tumor Area

\begin{tabular}{|c|c|c|}
\hline Patient ID & \% of Damage area & $\begin{array}{c}\text { \% of } \\
\text { Damage } \\
\text { area }\end{array}$ \\
\hline 11 & 4315 & $\begin{array}{c}17.2 \\
6\end{array}$ \\
\hline 21 & 1068 & 4.27 \\
\hline 31 & 225 & 1.74 \\
\hline 41 & 100 & 7.10 \\
\hline 51 & 1060 & 4.24 \\
\hline
\end{tabular}

We are representing the area of the tumor calculated previously with the help of the following graph.

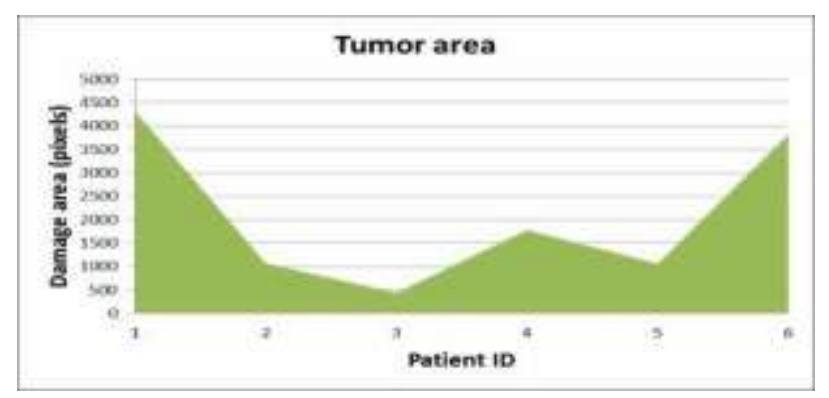

Graph 4.1: Histogram of possible tumour area

\section{CONCLUSION}

In this project, we present an algorithm to detect a special region from brain MRI. Brain MR Image is a complex system to be segmented with an efficient method to have variable type tissues. Determines whether an incoming brain MRI image represents a healthy brain or brain tumor. At first, the magnetic resonance of the brain of health has an obviously symmetrical character. However, if there is a macroscopic tumor, the characteristic of symmetry will weaken. We developed a segment algorithm to detect and extract the tumor region automatically.

\section{REFERENCES}

1.Kung-hao Liang and Tardi Tjahjadi, (January,2006).

"Adaptive Scale Fixing for Multi-scale Texture

Segmentation", IEEE Transactions on Image processing, Vol. 15, No.1, (pp.249-256).

2.Wiley Wang, et al. (July 2006). "Hierarchical Stochastic Image Grammars for Classification and Segmentation", IEEE Transactions on Image processing, Vol. 15, No.7, (pp. 3033-3052).

3.T.J.Davis and D.Gao, (1995). "Phase-contrast imaging of weakly absorbing materials using hard x-rays," Nature, Vol.373,(pp.595-597).

4. Jiao Feng and Fu Desheng, 2007, "Fast Gray-Contrast Enhancement of X-ray Imaging for Observing Tiny Characters", Proceedings of ICBBE, Vol.2, (pp.694-697).

5. Hongxia Yin, et al, 2005, "Diffraction Enhanced X-ray Imaging for Observing Guinea Pig Cochlea", Proceedings of the 2005 IEEE Engineering in Medicine and Biology 27th Annual Conference,(pp.5699-5701),

6. Kamber, M., Shingal, R., Collins, D., Francis, D., et al. 1995, "Model-based, 3-D segmentation of multiple sclerosis lesions in magnetic resonance brain images", IEEE-TMI, pp.442-453,

7.K.Selvanayaki, Dr. P.Kalugasalam, (February 2013).Intelligent Brain Tumor Tissue Segmentation From Magnetic Resonance Image (MRI) Using Meta Heuristic Algorithms Journal Of Global Research In Computer Science Volume 4, No. 2.

8. A. Sivaramakrishnan And Dr. M Karnan, (April 2013). "A Novel Based Approach For Extraction Of Brain Tumor In MRI Images Using Soft Computing Techniques", International Journal Of Advanced Research In Computer And Communication Engineering, Vol. 2, Issue 4.

9. Manoj K Kowar and Sourabh Yadav (April 2012).”Brain Tumor Detction and Segmentation Using Histogram Thresholding", International Journal of Engineering and Advanced Technology (IJEAT) ISSN: 2249 - 8958, Volume1 , Issue-4.

10. Sabina Breitenmoser, (21 February 2005), "Evaluation and implementation of neural brain activity detection methods for fMRI", Linkoping University, Sweden, pp.13-18.

11.Manoj K Kowar and Sourabh Yadav,(April 2012).’Brain Tumor Detction and Segmentation Using Histogram Thresholding" International Journal of Engineering and Advanced Technology (IJEAT) ISSN: 2249 - 8958, Volume1 , Issue-4. 
International Journal of Engineering Applied Sciences and Technology, 2019

Vol. 4, Issue 3, ISSN No. 2455-2143, Pages 244-248

Published Online July 2019 in IJEAST (http://www.ijeast.com)

12. Ola Friman, (2003)."Adaptive Analysis of Functional MRI Data", Dissertation No. 836 at Department of Biomedical Engineering, Linkoping University, Sweden, , (pp. 8-10).

13. Charutha S 1, M.J.Jayashree 2" (May 2014). An Integrated Brain Tumour Detection Technique" International Journal of Research in Advent Technology, Vol.2, No.5, E-ISSN: 23219637. 